\title{
ПРИМЕНЕНИЕ МНОГОЧЛЕНОВ БАТТЕРВОРТА ДЛЯ ПОСТРОЕНИЯ МОДАЛЬНЫХ ДИФФЕРЕНЦИАТОРОВ
}

\author{
(C) 2020 А. В. Дылевский \\ Воронежский государственный университет \\ Университетская пл., 1, 394018 Воронеж, Российская Федерация
}

\begin{abstract}
Аннотация. В статье рассматривается метод построения автоматических модальных дифференциаторов с помощью нормированных многочленов Баттерворта. Синтез модальных дифференциаторов сводится к построению следящей системы управления для объекта, представляющего собой последовательное соединение интегрирующих звеньев. Полюсы автоматических модальных дифференциаторов являются корнями многочленов Баттерворта. Полином Баттерворта представляет собой знаменатель фильтра Баттерворта. Корни многочлена Баттерворта расположены на круге некоторого радиуса равноудалённо друг от друга в левой полуплоскости комплексной плоскости. Радиус круга определяется частотой среза фильтра Баттерворта. Построенные модальные дифференциаторы осуществляют асимптотически точное помехозащищенное дифференцирование сигналов из достаточно широкого класса. Класс дифференцируемых сигналов определяется некоторым дифференциальным неравенством и содержит множество непрерывно-дифференцируемых функций с ограниченной старшей производной. Класс дифференцируемых сигналов включает логарифмические, экспоненциальные и тригонометрические функции, алгебраические многочлены. Следует отметить, что вышеперечисленными функциями класс дифференцируемых сигналов не исчерпывается. Модальные дифференциаторы являются помехозащищенными по отношению к высокочастотным помехам. Полоса пропускания сигналов может быть задана за счёт выбора соответствующей частоты среза фильтра Баттерворта. В статье проводится сравнительный анализ модальных дифференциаторов, построенных с помощью многочленов Баттерворта и дифференциаторов, полюсы которых образуют геометрическую последовательность. Для анализа дифференциаторов используются амплитудно-частотные и фазо-частотные характеристики. Приводится пример построения дифференциатора первого порядка. Во временной области рассматривается результат дифференцирования низкочастотного гармонического сигнала. Предлагаемые в статье дифференциаторы могут быть использованы для синтеза высококачественных систем автоматического управления, а также для решения широкого круга задач, связанных с автоматическим дифференцированием.
\end{abstract}

Ключевые слова: модальный дифференциатор, многочлены Баттерворта, класс сигналов, точность дифференцирования, помехозащищенность.

\section{ВВЕДЕНИЕ}

Одной из важнейших задач синтеза систем автоматического управления является получение информации о производных полезного сигнала [1-5]. В системах автоматического управления полезный сигнал, пред-

Дылевский А. В.

e-mail:nefta@yandex.ru ставляющий собой входное воздействие или выход объекта управления, заранее неизвестен, а выделен лишь класс таких сигналов [6]. Поэтому представляется вполне очевидной необходимость в использовании дифференцирующих устройств, осуществляющих многократное дифференцирование сигналов из некоторого класса.

Сложность решения задачи многократного дифференцирования объясняется принКонтент доступен под лицензией Creative Commons Attribution 4.0 License. The content is available under Creative Commons Attribution 4.0 License. 
ципиальной неосуществимостью точного дифференцирования из-за физической нереализуемости идеального дифференцирующего устройства с передаточной функцией $W(p)=p, p \in C$, а также присутствия высокочастотных аддитивных помех измерения полезного сигнала [6-8].

\section{АНАЛИЗ РЕЗУЛЬТАТОВ ПРЕДШЕСТВУЮЩИХ РАБОТ}

В [6] был предложен метод синтеза дифференциаторов, с помощью которых можно осуществлять многократное дифференцирование широкого класса сигналов в присутствие высокочастотных помех измерения. Основная идея метода заключается в том, что синтез дифференциаторов сводится к построению следящей системы, где в качестве объекта выступает цепочка интеграторов. Показано, что дифференциатор, полюсы передаточной функции которого расположены в левой полуплоскости комплексной плоскости, осуществляет асимптотически точное помехозащищенное дифференцирование сигналов достаточно широкого класса. Однако поведение ошибки дифференцирования во многом зависит от распределения этих полюсов на комплексной плоскости. В данной статье полюсы дифференциатора рассматривается как корни нормированных многочленов Баттерворта, определяющие одноимённые фильтры $[9,10]$.

\section{ПОСТАНОВКА ЗАДАЧИ}

Обозначим через $F_{m}$ множество всех функций $f \in C^{m}[0,+\infty), \quad$ удовлетворяющих $\forall M>0$ дифференциальному неравенству

$$
\left|f^{(m)}(t)\right| \leq M \quad \forall t \geq 0 .
$$

Класс сигналов $F_{m}$ является достаточно широким. Например, некоторые логарифмические, экспоненциальные и тригонометрические функции, алгебраические многочлены удовлетворяют требуемым условиям.

Требуется разработать метод построения передаточных функций дифференцирующих устройств, осуществляющих асимптотически точное помехозащищенное дифференцирование сигналов класса $F_{m}$.

\section{МЕТОДЫ И МАТЕРИАЛЫ ИССЛЕДОВАНИЯ (ТЕОРЕТИЧЕСКАЯ ЧАСТЬ)}

В [6] показано, что устройство с передаточной функцией

$$
W_{k}(p)=\frac{p^{k} L(p)}{D(p)}, \quad k=\overline{0, m-1},
$$

является дифференциатором $k$-го порядка сигналов из класса $F_{m}$. Здесь $D(p)$ - желаемый многочлен,

$$
\begin{gathered}
D(p)=\sum_{i=0}^{n} d_{i} p^{n-i}, d_{0}=1, n \geq 2 s, \forall s \geq m, \\
L(p)=\sum_{i=s}^{n} d_{i} p^{n-i} .
\end{gathered}
$$

Выберем желаемый многочлен $D(p)$ следующим образом:

$$
\begin{gathered}
D(p)=\prod_{j=1}^{n}\left(p+\lambda_{j}\right), \\
\operatorname{Re} \lambda_{j}>0 ; \lambda_{j} \neq \lambda_{i}, j \neq i, i, j=\overline{1, n} .
\end{gathered}
$$

Было показано [6], что устройство с передаточной функцией (2) при условиях (3)-(5) является асимптотическим (по $n$ и $t$ ) дифференциатором сигналов класса $F_{m}$, если значения $\lambda_{j}$ являются членами геометрической прогрессии

$$
\lambda_{j}=\beta q^{j-1}, \beta>0, q>1 .
$$

Таким образом, предлагаемый метод построения модальных дифференциаторов позволяет получать за счёт увеличения порядка передаточной функции дифференциатора, т. е. за счёт усложнения структуры дифференциатора, асимптотически точное значение производных любого сигнала из класса $F_{m}$. Кроме того, дифференциаторы, синтезированные на основе предлагаемого подхода, являются помехоустойчивыми по отношению к высокочастотным помехам, так как относительный порядок передаточной функции дифференциатора равен $s-k(k=\overline{0, m-1})$.

Следует отметить, что выбор корней желаемого многочлена $D(p)$ в виде геометрической прогрессии (6) не является единственно возможным. Например, в $[6,8]$ показано, что корни многочлена $D(p)$ могут являться членами арифметической прогрессии или быть 


\section{А. В. Дылевскици}

кратными. Однако в этом случае доказать асимптотическую точность дифференцирования по $n$, вообще говоря, не удаётся. Поэтому вызывают интерес другие возможные распределения корней желаемого многочлена. В теории фильтрации особое внимание уделяется фильтрам Баттерворта [11-15]. Это связано с тем, что амплитудно-частотная характеристика фильтра Баттерворта максимально гладкая на частотах полосы пропускания и снижается практически до нуля на частотах полосы подавления, т. е. амплитудная характеристика фильтра Баттерворта близка по форме к прямоугольной характеристике идеального фильтра.

Рассмотрим передаточную функцию фильтра Баттерворта $[9,10]$

$$
H(p)=\frac{G_{0}}{\prod_{k=1}^{n}\left(p-p_{k}\right) / \omega_{c}}=\frac{G_{0}}{B_{n}(p)} .
$$

Полюсы $p_{k}, k=\overline{1, n}$, передаточной функции фильтра Баттерворта расположены на круге радиуса $\omega_{c}\left(\omega_{c}-\right.$ частота среза) равноудалённо друг от друга в левой полуплоскости $p$-плоскости. Полюс $p_{k}$ определяется из следующего выражения:

$$
p_{k}=\omega_{c} e^{\frac{j(2 k+n-1) \pi}{2 n}}=\omega_{c} s_{k}, k=\overline{1, n} .
$$

В формуле (7) $G_{0}=$ const - коэффициент усиления на постоянной составляющей (коэффициент усиления на нулевой частоте). Действительно,

$$
H(0)=\frac{G_{0}}{\prod_{k=1}^{n}\left(-p_{k}\right) / \omega_{c}}=\frac{\left(-\omega_{c}\right)^{n} G_{0}}{\prod_{k=1}^{n} p_{k}} .
$$

В силу формулы (8) имеем

$$
\begin{gathered}
\prod_{k=1}^{n} p_{k}=\prod_{k=1}^{n} \omega_{c} \exp \left(\frac{j(2 k+n-1) \pi}{2 n}\right)= \\
=\omega_{c}^{n} \exp \left(\frac{j \pi}{2 n} \sum_{k=1}^{n}(2 k+n-1)\right)= \\
=\omega_{c}^{n} \exp (j \pi n)=\left(-\omega_{c}\right)^{n} .
\end{gathered}
$$

Таким образом, из равенства (9) получаем

$$
H(0)=G_{0} .
$$

Поэтому в дальнейшем без ограничения общности будем рассматривать случай

$$
G_{0}=1 \text {. }
$$

Знаменатель $B_{n}(p)$ передаточной функции фильтра Баттерворта называется полиномом Баттерворта. Как правило, полиномы Баттерворта $B_{n}(p)$ нормируются на частоте среза $\omega_{c}$ и рассматриваются нормированные полиномы Баттерворта $B_{n}^{*}(s)$

$$
\begin{gathered}
B_{n}(p)=\prod_{k=1}^{n}\left(p-p_{k}\right) / \omega_{c}= \\
=\prod_{k=1}^{n}\left(s-s_{k}\right)=B_{n}^{*}(s),
\end{gathered}
$$

где

$$
s=p / \omega_{c} .
$$

Полиномы Баттерворта $B_{n}^{*}(s)$ могут записываться в комплексной форме, как показано выше. Однако обычно полиномы Баттерворта записываются в виде соотношений с вещественными коэффициентами, т. е. комплексно-сопряжённые пары объединяются с помощью умножения. Нетрудно проверить, что нормированные полиномы Баттерворта $B_{n}^{*}(s)$ имеют следующую каноническую форму:

$$
\begin{gathered}
B_{n}^{*}(s)=\prod_{i=1}^{n / 2}\left(s^{2}+2 s \sin \frac{(2 i-1) \pi}{2 n}+1\right), \\
n-\text { четное, } \\
B_{n}^{*}(s)=(s+1) \times \\
\times \prod_{i=1}^{n / 2-1}\left(s^{2}+2 s \sin \frac{(2 i-1) \pi}{2 n}+1\right), \\
n-\text { нечетное. }
\end{gathered}
$$

Как было отмечено выше, амплитудно-частотная характеристика фильтра Баттерворта на частотах полосы пропускания близка по форме к прямоугольной характеристике идеального фильтра и снижается практически до нуля на частотах полосы подавления (см. рис. 1 при $\omega_{c}=1$ для $n=4$ и $n=9$ ).

Фазо-частотная характеристика фильтра Баттерворта на частотах полосы пропускания имеет линейный характер, что свидетельствует о запаздывающих свойствах таких фильтров. На рис. 2 представлены фазо-частотные характеристики фильтра (2) при следующих значениях: $k=0, s=m=2, n=4, \beta=1, q=1,1$ (кривая a) и фильтра Баттерворта (кривая b) при $n=4$. 


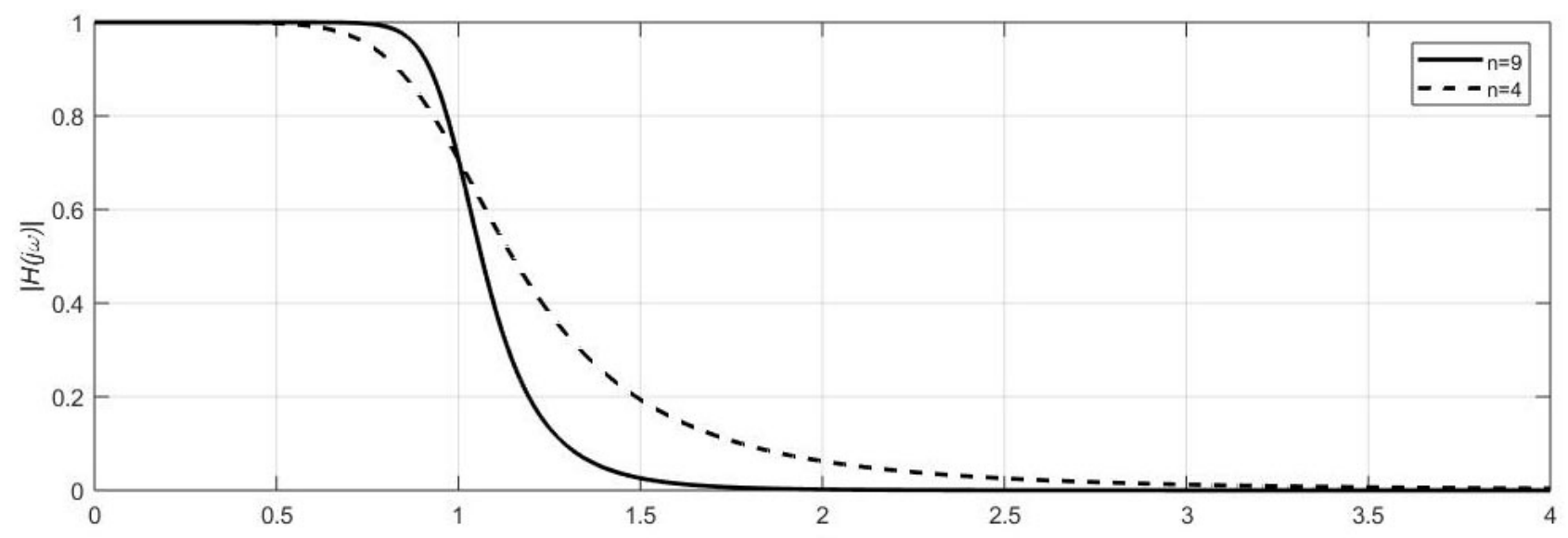

Рис. 1. Амплитудно-частотная характеристика фильтра Баттерворта

[Fig. 1. Amplitude-frequency response of the Butterworth filter]

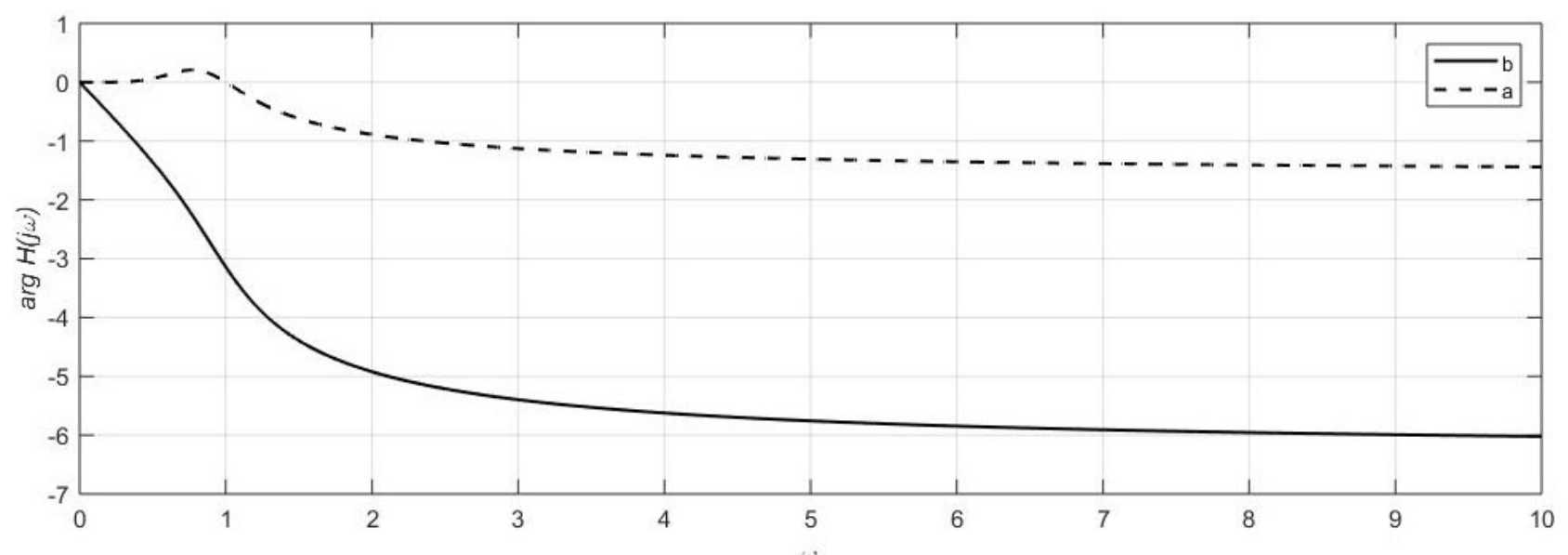

Рис. 2. Фазо-частотная характеристика фильтра Баттерворта

[Fig. 2. Phase-frequency response of the Butterworth filter]

\section{РЕЗУЛЬТАТЫ ИССЛЕДОВАНИЙ И ИХ ОБСУЖДЕНИЕ}

В передаточной функции дифференциатора (2) определим желаемый характеристический полином $D(p)$ как многочлен Баттерворта, т. е. положим

$$
D(p)=B_{n}(p) .
$$

Следует отметить, что многочлен $B_{n}(p)$ является устойчивым. В самом деле, согласно (8) имеем

$$
\begin{gathered}
\operatorname{Re} p_{k}=\omega_{c} \operatorname{Re} \exp \left(\frac{j(2 k+n-1) \pi}{2 n}\right)= \\
=-\omega_{c} \sin \frac{(2 k-1) \pi}{2 n}, \quad k=\overline{1, n} .
\end{gathered}
$$

В силу условия $k=\overline{1, n}$ выполняется неравенство $\frac{1}{2}<1 \leq k \leq n<n+\frac{1}{2}$. Поэтому $0<\frac{(2 k-1) \pi}{2 n}<\pi$ и $\sin \frac{(2 k-1) \pi}{2 n}>0$. Так как по построению $\omega_{c}>0$, то $\operatorname{Re} p_{k}<0, k=\overline{1, n}$.

В формуле (2) при условиях (3), (4) положим $s=m=2, k=1$ и $n=5$. Тогда передаточная функция дифференциатора имеет вид

$$
W_{B, 1}(p)=\frac{p L_{B}(p)}{B_{5}(p)},
$$

где

$$
\begin{gathered}
B_{5}(p)=p^{5}+3,23607 p^{4}+5,23607 p^{3}+ \\
+5,23607 p^{2}+3,23607 p+1 ; \\
p_{1}=-1, p_{2.3}=-0,8090 \pm 0,5878 j, \\
p_{4.5}=-0,3090 \pm 0,9511 j ; \\
L_{B}(p)=5,23607 p^{3}+5,23607 p^{2}+ \\
+3,23607 p+1 .
\end{gathered}
$$

Передаточную функцию модального дифференциатора определим при $s=m=2, k=1$, $n=5, \beta=1.2, q=1.1$. В этом случае имеем 


\section{А. В. Дылевскиц̆}

$$
W_{1}(p)=\frac{p L(p)}{D(p)},
$$

где

$$
\begin{gathered}
D(p)=p^{5}+7,32612 p^{4}+21,37175 p^{3}+ \\
+31,03179 p^{2}+22,42714 p+6,45406 \\
\lambda_{1}=1,2, \lambda_{2}=1,32, \lambda_{3}=1,452 \\
\lambda_{4}=1,5972, \lambda_{5}=1,75692 \\
L(p)=21,37175 p^{3}+31,03179 p^{2}+ \\
+22,42714 p+6,45406
\end{gathered}
$$

На рис. 3 изображены амплитудно-частотные характеристики модального дифференциатора (16) (кривая а), дифференциатора (15), построенного с помощью многочлена Баттерворта (кривая b), и идеального дифференциатора (кривая с). На и рис. 4 представлены фазо-частотные характеристики модального дифференциатора (16) (кривая а) и дифференциатора (15), построенного с помощью многочлена Баттерворта (кривая b).

Результат дифференцирования сигнала $f(t)=\sin 0,3 t$ представлен на рис. 5 . Кривая а соответствует производной $f^{\prime}(t)=0,3 \cos 0,3 t$, кривая $\mathrm{b}$ - результат дифференцирования c помощью модального дифференциатора (16), кривая с - результат дифференцирования дифференциатора (15), построенного с помощью полинома Баттерворта.

\section{ЗАКЛЮЧЕНИЕ}

В статье рассмотрен метод построения дифференциаторов с помощью нормированных многочленов Баттерворта. Несмотря на то, что фильтры Баттерворта обладают близкой к идеальной амплитудно-частотной характеристикой, линейность фазо-частот-

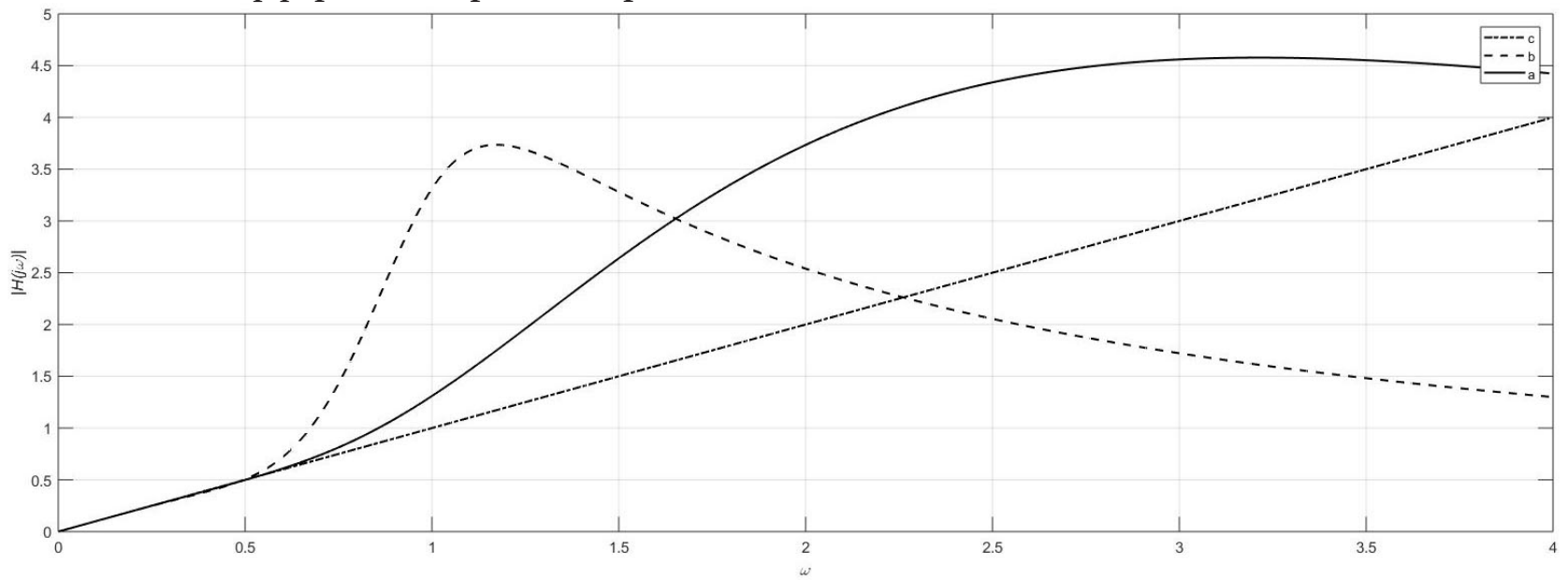

Рис. 3. Амплитудно-частотные характеристики дифференциаторов

[Fig. 3. Amplitude-frequency characteristics of differentiators]

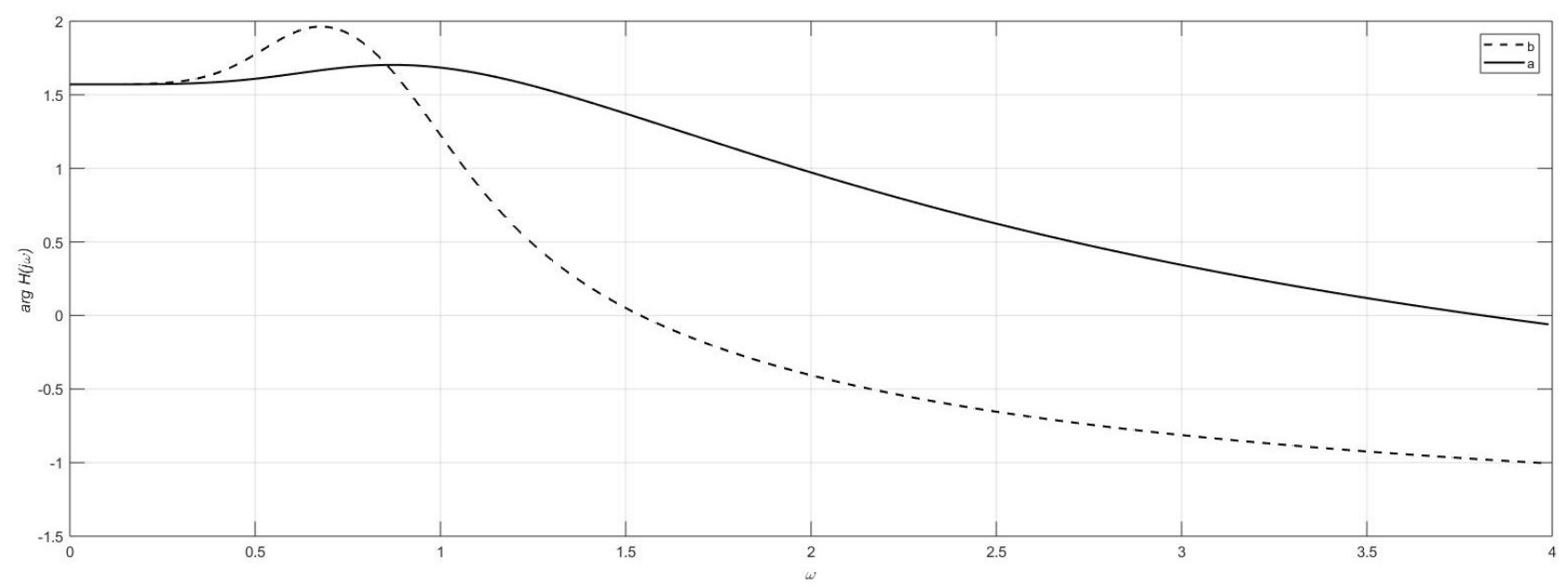

Рис. 4. Фазо-частотные характеристики дифференциаторов

[Fig. 4. Phase-frequency characteristics of the differentiators] 


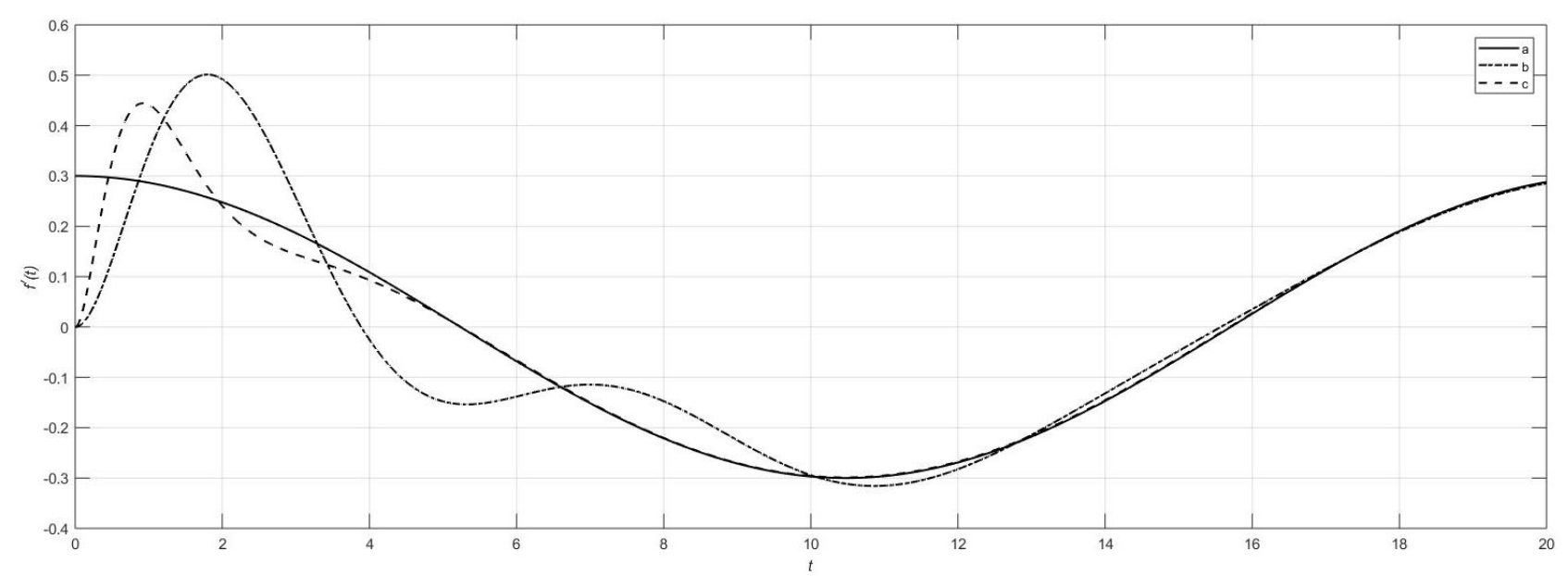

Рис. 5. Результат дифференцирования с помощью дифференииатора

[Fig. 5. Result of differentiation using a differentiator]

ной характеристики в полосе пропускания и наличие комплексно-сопряженных полюсов значительно ухудшают качество дифференцирования сигналов: наблюдаются эффекты запаздывания и колебательность. При этом выбор полюсов модального дифференциатора в виде геометрической прогрессии позволяет устранить эти недостатки.

\section{КОНФЛИКТ ИНТЕРЕСОВ}

Авторы декларируют отсутствие явных и потенциальных конфликтов интересов, связанных с публикацией настоящей статьи.

\section{СПИСОК ЛИТЕРАТУРЫ}

1. Гайдук, А. Р. Теория и методы аналитического синтеза систем автоматического управления (полиномиальный подход): монография / А. Р. Гайдук. - М. : Физматлит, 2018. - $360 \mathrm{c}$.

2. Подчукаев, В. А. Теория автоматического управления (аналитические методы): монография / В. А. Подчукаев. - М. : Физматлит, 2015. - 392 c.

3. Robust Adaptive Model Predictive Building Climate Control / M. Tanaskovic [и др.] // Proc. 20 World Congr. Int. Federat. Autom. Control (Toulouse, France, July 9-14, 2017). - Toulouse, 2017. - P. 1907-1912.

4. Sanz, R. Enhanced Disturbance Rejection for a Predictor-Based Control of LTI Systems with Input Delay / R. Sanz, P. Garcia, P. Albertos // Automatica. - 2016. - Vol. 72, P. 205-208.

5. Furtat, I. Disturbance Compensation with Finite Spectrum Assignment for Plants with Input Delay / I. Furtat., E. Fridman., A. Fradkov // IEEE Trans. Autom. Control. - 2018. - Vol. 63, No 1. - P. 298-305.

6. Лозгачев, Г. И. Автоматические дифференциаторы: построение и применение в задачах управления: монография / Г. И. Лозгачев, А. В. Дылевский. - Воронеж : Изд-во Воронеж. гос. ун-та, 2000. - 144 с.

7. Дылевский, А. В. Применение метода пространства состояний для синтеза дифференциаторов / А. В. Дылевский, Г. И. Лозгачев // Автоматика и телемеханика. - 1999. № 9. - C. 13-20.

8. Dylevskij, A. V. Modal differentiator design / A. V. Dylevskij, G. I. Lozgachev // Comput. Math. Model. - 2000. - Vol. 11, No 2. - P. 109118.

9. Smith, S. W. The Scientist and Engineer's Guide to Digital Signal Processing / S. W. Smith. San Diego: California Technical Publishing, 1997. $-626 \mathrm{p}$.

10. Лукас, В. А. Теория автоматического управления: учеб. для вузов. 2-е изд., перераб. и доп. / В. А. Лукас. - М.: Недра, 1990. - 416 с.

11. Bianchi, G. R. Sorrentino Electronic Filter: Simulation \& Design / G. Bianchi, R. Sorrentino. - New York: McGraw Hill, 2007. - 606 p.

12. Shen, T. Full-wave design of canonical waveguide filters by optimization / T. Shen [at al] // 


\title{
А. В. Дылевскиц̆
}

IEEE Transactions on Microwave Theory and Techniques. - 2003. - Vol. 51, No 2. - P. 504-511.

13. Lutovac, M. D. Filter Design for Signal Processing using MATLAB and Mathematica / M. D. Lutovac, D. V. Tosic, B. L. Evans. - New Jersey, USA.: Prentice Hall, 2001. - 756 p.
14. Rorabaugh, B. C. Approximation Methods for Electronic Filter Design / B. C. Rorabaugh. New York: McGraw-Hill, 1999. - 518 p.

15. Haykin, S. Adaptive Filter Theory/ S. Haykin. - Paramus, NJ : Prentice-Hall, 2001. - 936 p.

Дылевский А. В. - д-р техн. наук, доц., проф. кафедры ERP-систем и бизнес-процессов ф-та ПММ, Воронежский государственный университет. E-mail: nefta@yandex.ru

ORCID iD: https://orcid.org/0000-0003-1938-2419

DOI: https://doi.org/10.17308/sait.2020.1/2577

Received 23.01.2020

Accepted 15.03.2020

ISSN 1995-5499

\section{APPLICATION OF THE BUTTERWORTH POLYNOMIALS FOR THE DESIGN OF MODAL DIFFERENTIATORS}

\author{
(C) 2020 A. V. Dylevskij \\ Voronezh State University \\ 1, Universitetskaya Square., 394018 Voronezh, Russian Federation
}

\begin{abstract}
The article considers a method for designing automatic modal differentiators using normalized Butterworth polynomials. The synthesis of modal differentiators is reduced to the construction of a tracking control system for an object which is a series-connected integrating network. The poles of automatic modal differentiators are the roots of the Butterworth polynomials. The Butterworth polynomial is the denominator of the Butterworth filter. The roots of the Butterworth polynomial are located on a circle of a certain radius equidistant from each other in the left half plane of the complex plane. The radius of the circle is determined by the cutoff frequency of the Butterworth filter. The constructed modal differentiators provide asymptotically exact noise-immune differentiation of a fairly large class of signals. The class of differentiatable signals is defined by a differential inequality and contains a set of continuously differentiable functions with a bounded higher-order derivative. The class of differentiable signals includes logarithmic, exponential and trigonometric functions, and algebraic polynomials. It should be noted that the class of differentiable signals is not limited to the above functions. Modal differentiators are immune to high-frequency noise. The bandwidth of the signals can be set by choosing an appropriate cutoff frequency of the Butterworth filter. The article provides a comparative analysis of modal differentiators constructed using the Butterworth polynomials and differentiators whose poles form a geometric sequence. Amplitude-frequency and phase-frequency characteristics were used to analyse the differentiators. An example of constructing a first-order differentiator is given. The result of the differentiation of a low-frequency harmonic signal was considered in the time domain. The differentiators proposed in this article can be used for the synthesis of high-quality automatic control systems, as well as for solving a wide range of problems regarding automatic differentiation.

Keywords: modal differentiator, the Butterworth polynomials, signal class, differentiation accuracy, noise immunity.
\end{abstract}

Dylevskij A. V.

e-mail: nefta@yandex.ru 


\section{CONFLICT OF INTEREST}

The authors declare the absence of obvious and potential conflicts of interest related to the publication of this article.

\section{REFERENCES}

1. Gajduk A. R. Theory and methods of analytical synthesis of automatic control systems (polynomial approach). Moscow, Fizmatlit. 2018. $360 \mathrm{p}$.

2. Podchukaev V. A. Automatic control theory (analytical methods). Moscow, Fizmatlit. 2015. $392 \mathrm{p}$.

3. Tanaskovic M., Sturzenegger D., Smith R. and Morari M. Robust Adaptive Model Predictive Building Climate Control. Proc. 20 World Congr. Int. Federat. Autom. Control (Toulouse, France, July 9-14, 2017). Toulouse, 2017. P. 1907-1912.

4. Sanz R., Garcia P. and Albertos P. Enhanced Disturbance Rejection for a Predictor-Based Control of LTI Systems with Input Delay. Automatica. 2016. Vol. 72. P. 205-208.

5. Furtat I., Fridman E. and Fradkov A. Disturbance Compensation with Finite Spectrum Assignment for Plants with Input Delay. IEEE Trans. Autom. Control. 2018. Vol. 63. No 1. P. 298-305.

6. Lozgachev G. I. and Dylevskij A. V. Automatic differentiators: design and application in control tasks. Voronezh, Izd-vo Voronezh. gos. un-ta. 2000. 144 p.

7. Dylevskij A. V. and Lozgachev G. I. Application of the state space method for the synthesis of differentiators. Avtomatika i telemekhanika. 1999. No 9. P. 13-20.

8. Dylevskij A. V. and Lozgachev G. I. Modal differentiator design. Comput. Math. Model. 2000. Vol. 11. No 2. P. 109-118.

9. Smith S. W. The Scientist and Engineer's Guide to Digital Signal Processing. San Diego, California Technical Publishing, 1997.626 p.

10. Lukas V. A. Automatic control theory. Moscow, Nedra, 1990. 416 p.

11. Bianchi G. R. and Sorrentino R. Sorrentino Electronic Filter: Simulation \& Design. New York, McGraw Hill, 2007. 606 p.

12. Shen $T$ [at al] Full-wave design of canonical waveguide filters by optimization. IEEE Transactions on Microwave Theory and Techniques. 2003. Vol. 51. No 2. P. 504-511.

13. Lutovac M. and Tosic D. D. V. and Evans B. L. Filter Design for Signal Processing using MATLAB and Mathematica. New Jersey, USA, Prentice Hall, 2001. 756 p.

14. Rorabaugh B. C. Approximation Methods for Electronic Filter Design. New York, McGrawHill. 1999. 518 p.

15. Haykin S. Adaptive Filter Theory. Paramus, NJ, Prentice-Hall, 2001. 936 p.

Dylevskij A. V. - DSc in Technical Sciences, Associate Professor, Department of ERP-Systems and Business Processes, Faculty of Applied Mathematics, Informatics, and Mechanics, Voronezh State University.

E-mail: nefta@yandex.ru

ORCID iD: https://orcid.org/0000-0003-1938-2419 